\title{
Banco de Dados de Produtos não Madeireiros da Floresta Nacional do Tapirapé-Aquiri, Serra de Carajás
}

\author{
Luana do Carmi Oliveira Ferreira ${ }^{1}$, Alexandre Pereira da Costa Neto ${ }^{1}$, Gleysla Gonçalves de Carvalho Fernandes ${ }^{1}$, \\ Álisson Rangel Albuquerque ${ }^{1} \&$ André Luis Macedo Vieira ${ }^{2}$
}

Recebido em 25/11/2020- Aceito em 12/07/2021

\footnotetext{
${ }^{1}$ Universidade do Estado do Pará/UEPA, Brasil.<luana.itups@gmail.com, alexandreneto.exe@hotmail.com.br, gleysla14gg@gmail.com, alissonrangel@uepa.br>

${ }^{2}$ Instituto Chico Mendes de Conservação da Biodiversidade/ICMBio, Brasil. <andre.macedo@icmbio.gov.br>
}

\begin{abstract}
RESUMO - Conhecidos por suas diversas formas de utilização, os produtos florestais não madeireiros caracterizam-se como todo e qualquer produto de origem vegetal e arbórea, exceto madeira. A diversidade de usos desses produtos integra-se aos modos de vida de povos indígenas e de populações tradicionais que residem no interior de áreas florestais e rurais. O objetivo deste trabalho foi o desenvolvimento, a alimentação e a disponibilização de um banco de dados de produtos florestais não madeireiros da Floresta Nacional Tapirapé Aquiri (FLONATA). Para isso, utilizou-se de inventários florestais realizados na FLONATA, bem como de pesquisa bibliográfica em bases de dados digitais. Foram encontradas 182 espécies, pertencentes a 142 gêneros e a 61 famílias botânicas. Do total, 82 espécies apresentaram potenciais de uso não madeireiro e dessas $40 \%$ são utilizadas para fins alimentícios, como o fruto do cacau-do-mato (Theobroma speciosum). Cerca de $12 \%$ das espécies apresentaram uso medicinal, como a casca do breu-sucuúba (Protium amazonicum) e 19\% apresentam mais de um tipo de utilidade, como é o caso da bacaba (Oenocarpus bacaba). O banco de dados, em vias de ser disponibilizado, poderá ser utilizado pela comunidade para conhecer as espécies com potencial não madeireiro dentro da unidade de conservação, propiciando o desenvolvimento de ações conservacionistas, ecológicas e de cunho social.
\end{abstract}

Palavras-chaves: Banco de dados; produtos não madeireiros; uso sustentável.

\section{Database of non-Timber Products from the Tapirapé-Aquiri National Forest, Serra de Carajás}

ABSTRACT - Known for their various forms of use, non-timber forest products are characterized as any product of vegetal and arboreal origin, except wood. These products comprise the livelihoods of indigenous peoples and traditional populations that reside in the interior of forest and rural areas. The objective of this work was to develop, feed and make available a database of non-timber forest products from the Tapirapé Aquiri National Forest (FLONATA). For this, forest inventories carried out at FLONATA were used, as well as bibliographic research in digital databases. A total of 182 species were found, belonging to 142 genera and 61 botanical families. Of the total, 82 species had potential for non-timber use and of these, $40 \%$ are used for food purposes, such as the fruit of cocoa-domato (Theobroma speciosum). About $12 \%$ of the species had medicinal use, such as the bark of the succuúba pitch (Protium amazonicum) and 19\% had more than one type of utility, such as the bacaba (Oenocarpus bacaba). With the future completion of the database, it can be used by the community to learn about species with non-timber potential within the conservation unit, enabling the development of conservation, ecological and social actions.

Keywords: Database; non-wood products; sustainable use. 


\title{
Base de Datos de Productos no Maderables del Bosque Nacional Tapirapé- Aquiri, Serra de Carajás
}

\begin{abstract}
RESUMEN - Los productos forestales no maderables, conocidos por sus diversas formas de uso, se caracterizan como cualquier producto de origen vegetal y arbóreo, excepto la madera. Estos productos conforman las formas de vida de los pueblos indígenas y poblaciones tradicionales que residen en el interior de los bosques y áreas rurales. El objetivo de este trabajo fue el desarrollo, suministro y disponibilidad de una base de datos de productos forestales no maderables del Bosque Nacional Tapirapé Aquiri (FLONATA). Para ello, la creación de la base de datos se dividió en tres etapas: construcción, suministro y disponibilidad de información sobre la dinámica de estos productos. Se encontraron 182 especies, pertenecientes a 142 géneros y 61 familias botánicas. Del total, 82 especies mostraron potencial para uso no maderable y de estas, el $40 \%$ se utiliza con fines alimentarios, como el fruto del cacao-do-mato (Theobroma speciosum). Alrededor del 12\% de las especies tenían uso medicinal, como la corteza de bresucuúba (Protium amazonicum) y el 19\% tienen más de un tipo de utilidad, como la bacaba (Oenocarpus bacaba). Con la futura finalización de la base de datos, podrá ser utilizada por la comunidad para conocer y comprender los principales productos dentro de la unidad de conservación, posibilitando el desarrollo de acciones de conservación, ecológicas y sociales.
\end{abstract}

Palabras clave: Base de datos; productos forestales no maderables; uso sostenible.

\section{Introdução}

Classificada como a maior floresta tropical úmida da Terra, a Floresta Amazônica vem sendo considerada a região de maior biodiversidade de espécies animais e vegetais (Ter Steege et al., 2016, Piva et al., 2020). Segundo Matheus (2019) e Costa et al. (2020), essa região está localizada no centronorte do continente sul-americano, área geográfica onde se encontra o bioma amazônico. Para Alho (2012) e Mendoza et al. (2018), a variedade de seres vivos presentes na Amazônia não deve ser visualizada individualmente, e sim como um conjunto estrutural e funcional, na visão ecológica e no conceito de ecossistema.

Encontrados na floresta, os produtos florestais não madeireiros (PFNMs) são caracterizados como frutos, óleos, látex, sementes, cipós, resinas e outros (Balzon et al., 2004; Pinto et al., 2008). Para Ribeiro \& Ferreira (2018) e Santos et al. (2020), a diversidade encontrada na Amazônia é fonte de diversas espécies com potencial de uso medicinal, alimentício e outros. A variedade de produtos provenientes da floresta torna-se um suporte considerável para comunidades locais, como o extrativismo da castanha-do-pará pelos extrativista da RESEX Ouro Preto (Medeiros, 2018) e a utilização e comercialização da andiroba pela comunidade de Mangagal município em Igarapé-Miri, PA (Souza et al., 2019).

Localizada no bioma amazônico, no sudeste do estado do Pará, a Floresta Nacional do Tapirapé Aquiri (FLONATA) é uma unidade de conservação de uso sustentável e compõe um conjunto de áreas protegidas na Serra dos Carajás. Criada pelo Decreto n 97.720, de 05/05/1989 (Brasil, 1989), a FLONATA prevê no seu plano de manejo a utilização dos produtos florestais não madeireiros, além disso, na UC são desenvolvidas atividades de lavra mineral (IBAMA, 2006), educação ambiental, bem como a agricultura e o manejo de sistemas agroflorestais no entorno da unidade (Vieira et al., 2017).

Na FLONATA, o extrativismo é realizado pela Cooperativa de Extrativistas de Carajás (COEX), que coleta a folha-do-jaborandi (Pilocarpus macrophyllus Stapf ex Hom) e sementes nativas, como a favade-paca (Stryphnodendron pulcherrimum Willd. Hochr), propiciando o armazenamento, bem como o beneficiamento desses produtos e sua posterior comercialização. Atualmente 40 cooperados realizam o trabalho de coleta e fornecimento da folha, para a indústria farmacêutica que extrai diferentes substâncias para usos medicinal e estético.

Com o intuito de organizar informações sobre os produtos florestais não madeireiros da FLONATA e maximizar a organização de dados e informações sobre PFNMS, optou-se pelo uso de um Banco de dados (BD). Conforme Silva (2001) e Silva (2019), $\mathrm{BD}$ é uma coleção de dados relacionados entre si, organizados e armazenados de forma a possibilitar e facilitar a visualização de uma informação. Para Hilbert \& López (2011), Couto (2017) e Freitas et al. (2020), um BD permite a transformação de dados em informações a partir da segurança, eficiência, hospedagem e consulta por outros usuários. 
Sendo assim, neste trabalho, relatamos o desenvolvimento de um banco de dados interativo sobre os produtos florestais não madeireiros da Floresta Nacional Tapirapé Aquiri. Este projeto tem caráter ecológico, social e conservacionista, uma vez que o conhecimento levantado, documentado e disponibilizado contribuirá para a ampliação do conhecimento acerca dos PFNMs e para geração de renda das comunidades e dos extrativistas que vivem no entorno da FLONATA.

\section{Material e Métodos \\ Objeto de estudo}

A Floresta Nacional de Tapirapé Aquiri perfaz um perímetro total aproximado de $298.300 \mathrm{~m}$ em uma área total aproximada de 190.000ha. Protege várias tipologias florestais, destacandose a associação de Floresta Ombrófila Aberta Submontana com Palmeiras, Floresta Ombrófila Densa Submontana e Floresta Ombrófila Aluvial, que cobrem mais de $50 \%$ da FLONATA (ICMBio, 2006). A FLONATA está localizada nos municípios de São Félix do Xingu e Marabá, no sudeste do estado do Pará, e integra o complexo regional de unidades de conservação formado por unidades de conservação de proteção integral e uso sustentável e uma Terra indígena (Théry \& Théry, 2009).

\section{Levantamento de dados}

Para coleta de dados de espécies fornecedoras de produtos não madeireiras, utilizouse o resultado de um inventário florestal realizado no ano de 2014 em área de operação do Projeto Salobo em Marabá, Pará. Neste estudo, as unidades amostrais foram alocadas sistematicamente $e$ ao todo foram instaladas 52 amostras, perfazendo um total de 13 hectares amostrados. A identificação das espécies florestais teve o auxilio de mateiros, parabotânicos, especialistas, e literatura específica (Engenharia de Projetos Ltda, 2014).

Posteriormente à identificação das espécies não madeireiras realizada por meio desse inventário, criou-se um banco de dados interativo. A alimentação do banco de dados foi efetivada mediante a pesquisa bibliográfica. As plataformas digitais utilizadas compreendem o Reflora 2020, Scielo e Google acadêmico (Norder et al., 2019; Reflora, 2019; Rocha et al., 2020).

\section{Desenvolvimento do banco de dados}

A Figura 1 exemplifica o esquema do banco de dados desenhado para o armazenamento de informações referentes aos PFNMs da FLONATA. O software para o banco de dados utilizado neste estudo foi o MySQL que, por sua vez, é um Sistema de Gerenciamento de Banco de Dados (SGBD) livre e de código aberto (Souza \& Oliveira, 2019) e compatível com a maioria dos sistemas operacionais (Silva, 2001; Souza \& Oliveira, 2019). O banco de dados dos produtos florestais não madeireiros da FLONATA possui a linguagem de consulta SQL (Structured Query Language) (Park 2009) e a utilização de programas como o WAMPSERVER 64 (Romain Boursoun 2019) e o VISUAL STUDIO C+ (Microsoft 2019), que permitem a instalação do $\mathrm{BD}$ e o seu funcionamento.

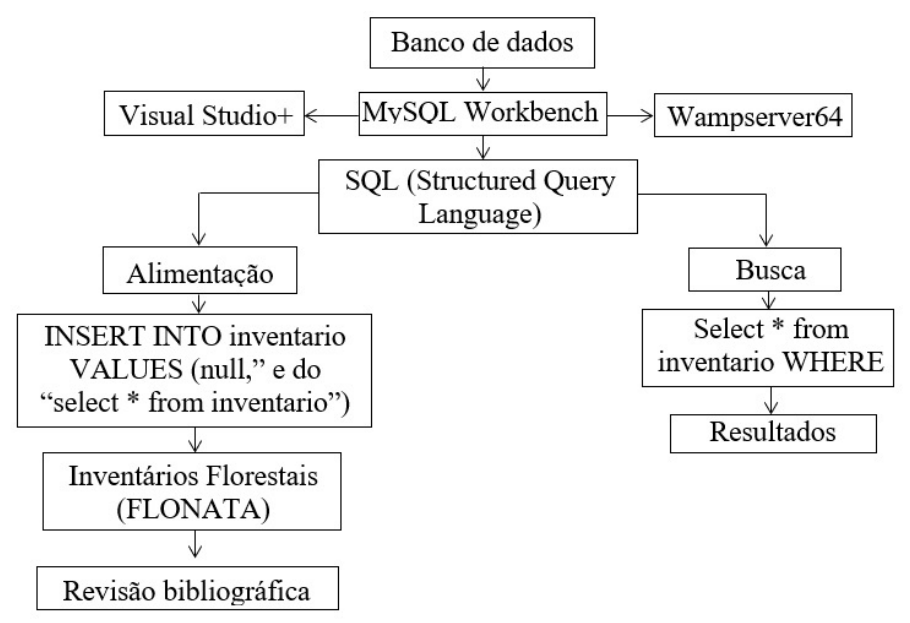

Figura 1 - Esquema do banco de dados dos produtos florestais não madeireiros da Floresta Nacional do Tapirapé Aquiri. Fonte: autor, 2019. 


\section{Resultados}

Das 188 espécies levantadas a partir dos inventários florestais realizados na Floresta Nacional do Tapirapé Aquiri em busca de informações sobre os produtos florestais não madeireiros, foram identificados 142 gêneros e 61 famílias botânicas.

Nessa identificação de famílias, observou-se que as espécies da familia Fabaceae apresentaram-se em um total de $45 \%$ dos indivíduos, como é o caso da fava-arara-tucupi (Parkia paraensis Ducke) e favabolota (Parkia pendula (Willd.). Walp.); seguido da família Moraceae (9\%), com mamica-de-cadela (Brosimum gaudichaudii Trécul.) e o amapá-doce (Brosimum parinarioides Ducke); Sapotaceae (9\%), com o abiu-branco (Pouteria guianensis
Aubl.) e abiu-caramuri (Pouteria manaosensis (Aubrév. \& Pellegr.); Burseraceae (8\%), com breuvermelho (Protium decandrum Aubl.) e breu-barote (Protium subserratum (Engl.) Engl.), entre outras que demostraram poucos indivíduos por família.

A forma de vida também foi um requisito a ser relevado em função dos produtos florestais não madeireiros. Das espécies amostradas, $84 \%$ têm habito de vida arbóreo, $8 \%$ caracterizam-se como arbustivo, 3\% como cipó, 3\% como palmeira e $2 \%$ como epífita. Em relação à ocorrência das espécies, destaca-se a região Norte do país, com $36 \%$ dos indivíduos presentes no inventário florestal, a região Centro-Oeste vem em seguida com $24 \%$, depois a Nordeste com $22 \%$, por fim, a Sudeste com $11 \%$ e a Sul com $6 \%$.

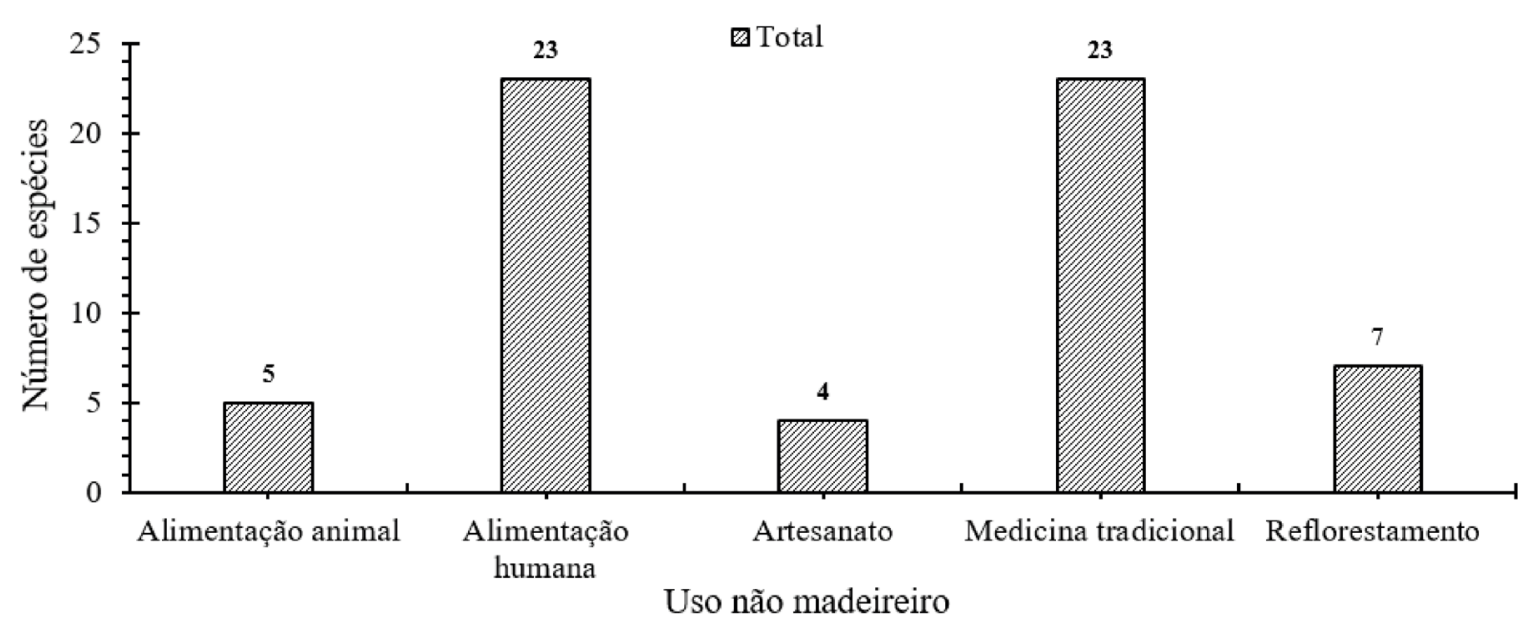

Figura 2 - Gráfico com o percentual da distribuição dos produtos não madeireiros.

O gráfico acima (Fig. 2) demostra que entre as principais formas de utilização estão o uso de folhas, frutos, cascas, sementes, entre outros. Em meio às espécies levantadas, apenas 82 apresentaram potencial não madeireiro conhecido, o que significa uma grande diferença quando comparado ao número de espécies, e uma carência de informações relacionadas aos produtos florestais não madeireiros.

Entre os usos, houve destaque também para os produtos florestais não madeireiros destinados ao uso alimentício (Fig. 2), com 23espécies, e tendo como parte utilizada os frutos, tanto para o consumo humano, como o cacaudo-mato (Theobroma speciosum Willd ex Spreng) e o cumaru (Dipteryx odorata (Aubl.) Willd)
(Carvalho, 2009), quanto para o consumo animal, como o abiu-branco (Pouteria guianensis Aubl.) e a melancieira (Alexa grandiflora Ducke) (Corrêa, 2015).

Foram encontradas 23 espécies indicadas para o uso medicinal (Fig. 2) entre os indivíduos analisados. Entre essas espécies, destacam-se o breu-amarelo (Protium paniculatum Engl.), cuja casca é utilizada na medicina tradicional para tratamentos fitoterápicos (Zoghbi, 1994), o bre-sucuúba (Protium amazonicum Cuatrec.), cujo óleo-resina é extraído para tratamentos cicatrizantes (Silva, 2006) e o jatobá (Hymenaea courbaril L.) que, por sua vez, é utilizado para tratamentos relacionados ao estômago através de suas folhas e frutos (Costa, 2012). 
Das espécies inseridas no banco de dados de produtos florestais não madeireiros da FLONATA, 7 são recomendadas para reflorestamento através de suas sementes como, por exemplo, o abiucutite (Pouteria macrophylla (Lam.) Eyma.) (INPA, 2019). Outras espécies são recomendadas para extração de óleos essenciais, como casca-preciosa (Aniba canelilla (H.B.K) Mez.) de onde se extrai o óleo que serve para inibir corrosão de açocarbono (Barros et al., 2015), ou possuem algum tipo de extrativo de interesse comercial ou para rituais étnicos.

Para as espécies estudadas, 8 espécies apresentam mais de um uso específico, como é o caso do babaçu (Attalea speciosa Mart) cujos fruto, folhas, palmito e óleo podem ser utilizados (INPA, 2019), a bacaba (Oenocarpus bacaba Mart), de onde se aproveitam o óleo, a palha, o fruto e o palmito (Ferreira, 2005) e o juazeiro (Zizyphus joazeiro Mart) cujos frutos e cascas são também utilizados para consumo humano (Carvalho, 2007).

Tabela 1 - Frequência absoluta e possibilidade de uso para as espécies com produtos não madeireiros da Floresta Nacional do Tapirapé Aquiri

\begin{tabular}{|l|l|l|l|}
\hline Nome vernacular & Nome científico & FA & PFNM \\
\hline Breu-amarelo & $\begin{array}{l}\text { Protium } \\
\text { heptaphyllum }\end{array}$ & 61,54 & SIM \\
\hline Breu-barrote & $\begin{array}{l}\text { Protium } \\
\text { subserratum }\end{array}$ & 40,38 & SIM \\
\hline Mata-matá-branco & $\begin{array}{l}\text { Eschweilera } \\
\text { coriacea }\end{array}$ & 36,54 & NÃO \\
\hline Paricá-de-espinho & $\begin{array}{l}\text { Acacia polyphylla } \\
\text { Cenostigma }\end{array}$ & 36,54 & NÃO \\
\hline Pau-preto & $\begin{array}{l}\text { tocatinum } \\
\text { Canela-de-jacamim }\end{array}$ & $\begin{array}{l}\text { Rinorea } \\
\text { guianensis }\end{array}$ & SIM \\
\hline Ingá-cipó & $\begin{array}{l}\text { Inga edulis } \\
\text { Protium } \\
\text { decandrum }\end{array}$ & 28,85 & NÃO \\
\hline Breu-vermelho & SIM \\
\hline Muiratinga-amarela & Astronium gracile & 25,00 & NÃO \\
\hline João-mole-da-folha- & Neea oppositifolia & 25,00 & NÃO \\
\hline -média
\end{tabular}

FA: Frequência Absoluta de espécies potencialmente não madeireiras, PFNM: Produto florestal não madeireiro.

Fonte: Coleta de dados do inventário florestal da Engenharia de Projetos Ltda (2014).
A Tabela 1 lista as espécies que se sobressaíram na análise de frequência absoluta no inventário florestal realizado na FLONATA (Engenharia de Projetos Ltda., 2014), bem como a existência, ou não, de um produto não madeireiro a ela associado. A frequência absoluta refere-se à porcentagem de subparcelas em que ocorre determinada espécie (Carvalho, 1984). As espécies fontes de PFNMS listadas na Tabela 1 fornecem um dos vários parâmetros fitossociológico que possibilitem o manejo sustentável desses produtos na Floresta Nacional do Tapirapé Aquiri.

Das espécies que possuem potencial de utilização (Tabela 1), destacam-se o breu-amarelo (Protium heptaphyllum Aubl.), sendo bastante utilizado na medicina tradicional por seu óleo (Mobin et al., 2016); o breu-barrote (Protium subserratum Aubl.) (Martão, 2013), cuja resina é bastante utilizada por comunidades amazônicas; o pau-preto (Cenostigma tocatinum Ducke), com sementes utilizadas em ações de reflorestamento (Rodrigues et al., 2020) e o ingá-cipó (Inga edulis Mart.), com frutos utilizados na alimentação humana (Falcão \& Clement, 2000).

\section{Discussão}

Os resultados apresentados neste estudo demostram que espécies levantadas a partir do inventário florestal auxiliam o dimensionamento e a capacidade da floresta em fornecer produtos naturais e utilizáveis. Segundo Lima et al. (2020), entende-se o inventário florestal como a enumeração da abundância e da distribuição de populações florestais. Entre os usos não madeireiros das espécies analisadas na FLONATA, houve destaque para o potencial alimentício a partir dos frutos de algumas espécies, como são os casos do bacupari (Rheedia gardneriana Planch. \& Triana) (Pinto, 2014) e do inajá (Maximiliana maripa) (Bezerra, 2011).

De acordo com Silva (2014) e Silva et al. (2017), a utilização dos múltiplos recursos não madeireiros frente a outras atividades, como a pecuária $e$ atividade madeireira, parece ser a forma mais viável para associar o desenvolvimento à manutenção da cobertura florestal. Em complemento, Silva et al. (2017) relatam que o potencial de mercado dos produtos florestais não madeireiros vem crescendo em função da valorização das florestas e dos serviços ecossistêmicos promovidos pelas mesmas. 
Na Amazônia, o açaí (Euterpe olearaceae Mart) destaca-se como um dos produtos florestais não madeireiros que possui um grande potencial agronômico, tecnológico, nutricional e econômico (Yuyama et al., 2011, Ximenes et al., 2016). Outras espécies essenciais para a região é a copaíba (Copaifera spp) (Pieri et al., 2009) e o cumaru (Dipteryx odorata) (Cabral, 2014), ambos utilizados na medicina tradicional.

Na FLONATA, entre as espécies de maior importância, destaca-se a castanheira (Bertholletia excelsa Bonpl.) (Silveira et al., 2016). No interior da unidade de conservação, a coleta da castanhado-pará é uma atividade frequentemente realizada pelos índios da TI Xikrin do Cateté (Silveira et al., 2015), há também a coleta e a comercialização dessa amêndoa por agricultores no entorno da unidade (Silva et al., 2017). Em associação com a Floresta Nacional de Carajás, na região ocorre a coleta da folha-do-jaborandi (Phillocarphus macrophillus) e de sementes nativas. Segundo Costa et al. (2016), desde 2012, os extrativistas são representados pela Cooperativa de Extrativistas de Carajás (COEX).

Rolim et al. (2006) relatam que o conjunto de unidades de conservação de Carajás ligado às possibilidades de cada unidade potencializa o uso racional dos recursos naturais, como o extrativismo vegetal, manejo florestal e conservação da biodiversidade. Silva et al. (2017), em seu estudo sobre aspectos socioambientais das propriedades no entorno da FLONATA, relatam que são necessárias atividades de extensão que auxiliem a agroecologia de famílias da região. Predozo et al. (2011) e Viana et al. (2016) enfatizam que são necessárias informações sobre a ecologia, uso e manejo desses produtos em prol de serem utilizados também como produto de troca em uma economia informal pelas comunidades locais.

Um banco de dados encaixa-se bem na necessidade de se promover uma organização do estoque de recursos florestais. A organização ocasionada pelo uso de um banco de dados desenvolve responsavelmente o armazenamento de informações de forma segura e eficaz. Ferreira et al. (2016) propõem o uso de um banco de dados MySQL para técnicas laboratoriais relacionadas ao ensino de química em universidades. Bertolini et al. (2019) utilizam o banco de dados MySQL para o armazenamento de índices de vegetação gerados para o monitoramento de pastagens. De acordo com Ferrari (2020), ferramentas computacionais podem ser utilizadas na construção e implantação um banco de dados biológico para armazenamento de informações etnobotânicas $e$ da sociobiodiversidade.

Assim, com o intuito de resolver essas necessidades, surgiram e surgem cada vez mais novas técnicas de implantação de bancos de dados, visando facilitar a vida do desenvolvedor e satisfazer as exigências do usuário (Oliveira, 2018). Acoplar dados e atualizar informações sobre as espécies com potencial não madeireiro da FLONATA é um caminho viável para a promoção do uso sustentável em um ecossistema natural em associação com o tripé social-econômicoambiental (triple bottom line) (Borges et al., 2013).

\section{Conclusão}

A criação, alimentação e publicação do banco de dados digitais interativo é uma etapa necessária para a organização de informações referentes aos produtos florestais não madeireiros dentro da Floresta Nacional do Tapirapé Aquiri. Com a futura finalização do banco de dados, ele estará online para futuras consultas e poderá ser utilizado pela comunidade para conhecer $e$ compreender os principais produtos dentro da unidade de conservação, gerando conhecimento e subsídios para o manejo florestal não madeireiro na unidade de conservação.

\section{Referências}

Alcântara GA. Caracterização farmacognóstica e atividade antimicrobiana da folha e casca do caule da Myrcia rostrata DC. Dissertação (Mestrado em Ciências Farmacêuticas) Universidade Federal de Goiás, 2012.

Alho CJR. Importância da biodiversidade para a saúde humana: uma perspectiva ecológica. Estudos avançados, 26 (74): 156-164, 2012.

Balzon DR, Silva JCGL, Santos AJ. Aspectos mercadológicos de produtos florestais não madeireiros análise retrospectiva. Floresta, 34 (3): 363-371, 2004.

Barros D et al. Casca Preciosa (Aniba canelilla) como Inibidor de Corrosão do Aço Carbono. Revista Virtual de Química, 7(5): 1743-1755, 2015.

Brasil. Decreto n 97.720, de 5 de maio de 1989. Diário Oficial da União. < http://legis.senado.leg.br/ norma/520360/publicacao/15802334>. Acesso em: $15 / 06 / 2019$ 
Bertolini CA, Tech ARB, Perreira LET. 2019. Desenvolvimento de software para aquisição de índices de vegetação: aplicações no monitoramento de pastagens, p. 21. In: IV Simpósio de Pós-Graduação em Zootecnia. Edição Especial. 2019.

Bezerra VS. O Inajá (Maximiliana maripa (Aubl.) Drude) como fonte alimentar e oleaginosa. Comunicado técnico. Embrapa Amapá, 2011.

Borges C, Borges MM, Ferreira VDRS, Najberg E, Tete MF. Empreendedorismo sustentável: proposição de uma tipologia e sugestões de pesquisa. Revista de Empreendedorismo e Gestão de Pequenas Empresas, 2 (1): 77-100, 2013.

Cabral DLDV. 2014. Potencial antimicrobiano de plantas da caatinga utilizadas na medicina tradicional como anti-inflamatórias. Tese (Doutorado em Ciências farmacêuticas). Universidade Federal de Pernambuco.

Carvalho JOP. Manejo de regeneração natural de espécies florestais. Comunicado técnico 296. Embrapa Amazônia Ocidental,1984. 22p.

Carvalho PER. Cumaru-Ferro Dipteryx odorata. Comunicado técnico 225. Embrapa Florestas, 23, 2009.

Carvalho PER. Juazeiro-Ziziphus joazeiro. Comunicado técnico. Embrapa Florestas, 2007.

Costa KJA et al. Aspectos fenológicos do açoita-cavaloLuehea speciosa Willd. (Malvaceae). In: Seminário de Iniciação Científica da Embrapa Amazônia Oriental p.21., 2017, Belém, PA.

Costa GM, Pimentel HVC, Cavalcante MMA. Implicações da implantação de usinas hidrelétricas $e$ unidades de conservação na hidrográfica do rio amazonas. Revista Equador, 9 (3): 233-251, 2020.

Costa MPD. 2012. Atividade biológica da seiva e de compostos extraídos da seiva de Hymenaea courbaril sobre leveduras e fungos filamentosos. Dissertação (Mestrado em Medicina Tropical e Saúde Pública). Universidade Federal de Goiás, 2012.

Couto RS. Estruturação, modelagem e implementação de banco de dados geoespaciais para o cadastro ambiental rural. Dissertação (Mestrado em Geociências Aplicadas). Universidade de Brasília, 2017.

Cruz ED. Germinação de sementes de espécies amazônicas: acariquara (Minquartia guianensis Aubl.). Comunicado técnico 296. Embrapa Amazônia Ocidental, 2010.

Elias GA, Santos R. Produtos florestais não madeireiros e valor potencial de exploração sustentável da Floresta Atlântica no Sul de Santa Catarina. Ciência Florestal, 26(1): 249-262, 2016.
Falcão MA, Lieras E. Aspectos fenológicos, ecológicos e de produtividade do mapati (Pourouma crecopiifolia Mart.). Acta Amazônica, 10: 711-724, 1980.

Falcão MDA, Clement CR. Fenologia e produtividade do Infá-Cipó (Inga edulis) na Amazônia Central. Acta amazônica, 30, 173-180, 2020.

Ferrari PA. 2020. Banco de dados etnobotânicos: construção de uma ferramenta de armazenamento $e$ proteção de informações sobre a sociobiodiversidade. TCC (Graduação em Ciências Biológicas). Universidade Federal de Santa Cantarina.

Ferreira, M. Bacaba (Oenocarpus bacaba Mart.). Comunicado técnico. Embrapa Rondônia, 2005.

Guerra FGPQ, Santos AJ, Sanquetta CR, Bittencourt AM, Almeida NA. Quantificação e valoração de produtos florestais não-madeireiros. Floresta, 39(2): 431-439. 2008.

Hilbert M, López P. The Worlds Technological Capacity. Science, 332: 60 - 65, 2011.

Homma, A. 2014. Extrativismo vegetal na Amazônia: história, ecologia, economia e domesticação. Brasilia, DF: Embrapa Amazônia Oriental - Livro científico. 468p.

IBAMA (Instituto Brasileiro do Meio Ambiente e dos Recursos Naturais Renováveis). Plano de Manejo para Uso Múltiplo da Floresta Nacional do Tapirapé-Aquiri. 2006. < http://www.ibama.gov.br/index.php>. Acesso em: $23 / 10 / 2018$

ICMBio (Instituto Chico Mendes de Conservação da Biodiversidade). 2006. Plano de manejo para uso múltiplo da Floresta Nacional do Tapirapé Aquiri. $<$ http://www.icmbio.gov.br/portal/images/stories/imgsunidades-coservacao/flona_tapirape-aquiri.pdf $>$. Acesso em: 25/10/2018.

INPA (Instituto Nacional de Pesquisas Amazônicas). 2019. Lista das Espécies Florestais e Arbustivas de Interesse Econômico na Amazônia Ocidental. Disponível em: $<$ https://www.inpa.gov.br/sementes/ arquivos/tabela_Nome_Cientifico_Usos.pdf. $>$ Acesso em: 04/01/2019.

Kruel VTF, Peixoto AL. Etnobotânica na Reserva Extrativista Marinha de Arraial do Cabo, RJ, Brasil. Acta Botânica Brasílica, 18(1), 2004.

Lima RB, Ferreira RLC, Silva JAA, Guedes MC, Silva DAS, Oliveira CP. Valoração de componentes não madeireiros na Amazônia: metodologias de quantificação para a geração de renda. Brazilian Applied Science Review, 4 (2): 561-591, 2020.

Magalhães, LM. Identificação de flavonoides por LCMS/MS no extrato de folhas da Trattinnickia rhoifolia (Willd) e avaliação da atividade antioxidante. $<$ https:// ppbio.inpa.gov.br/sites/default/files/Magalhaes_L_M_ Dissertacao_2017.pdf. > Acesso em 12/11/2019. 
Mendoza, AY, Santana RS, Santos VS, Lima RA. Diversidade de Basidiomycota na Reserva Natural Palmari, Amazonas, Brasil. Gestão e Sustentabilidade Ambiental, 7(4): 324-340, 2018.

Mateus ACC. Os conhecimentos tradicionais associados à biodiversidade amazônica: proteção jurídica $e$ sustentabilidade. Ponto de vista Jurídico, 8 (2), 2019.

Martão VM. 2013. Atividade antioxidante in vitro de plantas medicinais da Amazônia Ocidental. Dissertação (Mestrado em Desenvolvimento Regional e Meio Ambiente). Universidade Federal de Rondônia.

Mobin M. Análise MDGC-MS de óleos essenciais de Protium heptaphyllum (Aubl.) e sua atividade antifúngica contra a espécie cândida. Revista Brasileira de Plantas Medicinais, 18, 531-538, 2016.

MySQL. MySQL 8.0 Reference Manual. 2018.<https:// dev.mysql.com/doc/refman/8.0/en/what-is-mysql. html. > Acesso em: 10/11/2018.

Norder LA. Agroecologia em terras indígenas no Brasil: uma revisão bibliográfica. Espaço Ameríndio, 13(2): 291-329, 2019.

Oliveira MS, Melo NF, dos Santos LC, de Oliveira WP. Banco de dados No-SQL X Banco de dados SQL South American Development Society Journal, 4 (11): 298, 2018.

Palheta RA, Morais VMC, Furtado ACS, Souza PR, Vieira WO. Extrativismo de produtos não madeireiros: um estudo de caso na comunidade quilombola Menino Jesus - PA. In VII Simpósio de Ciências e Estudos Ambientais da Amazônia, Belém. 2017

Pereira CMS, Assis WS, Araújo CS. O manejo da Andiroba e a contribuição para a preservação ambiental: o caso do Grupo de Trabalhadoras Artesanais e Extrativistas (GTAE) do Projeto de Assentamento Agroextrativista Praialta Piranheira (PAE)-PA. Cadernos de Agroecologia, 10 (3), 2016.

Perreira MRN, Tonini H. Fenologia da Andiroba (Carapa guianensis Aubl.) no sul do estado de Roraima. Ciência Florestal, 22(1): 47-58, 2012.

Piva LRO, Sanquetta CR, Wojciechowski J, Corte, AP. Fitossociologia em comunidades florestais do projeto RadamBrasil no Bioma Amazônia. Biofix Scientific Journal, 5(2): 264-271, 2020.

Pinto PM., 2013. Pós-colheita de abiu, bacupari e camu-camu, nativos da Região Amazônica, cultivados no Estado de São Paulo. Tese (Doutorado e Fitotecnia). Universidade de São Paulo.

Pieri FA, Mussi MC, Moreira MAS. Óleo de copaíba (Copaifera sp.): histórico, extração, aplicações industriais e propriedades medicinais. Revista Brasileira de Plantas Medicinais, 11, 465-472, 2009.
Prospera ETP., 2010. Caracterização da fruta do Jacaratia spinosa e processamento do doce de jaracatiá em calda com avaliação da estabilidade. Dissertação (Mestrado em Ciência e Tecnologia de Alimentos). Universidade de São Paulo 139p.

Predozo EA, Silva TN, SatoSAS, Oliveira NDA. Produtos Florestais Não Madeiráveis (PFNMS): as Filières do Açaí e da Castanha da Amazônia. Administração e Negócios da Amazônia-RARA, 3(2): 88-112, 2011.

Queiroz JAL, Machado AS, Husokawa RT, Silva IC. Estrutura e Dinâmica de floresta de várzea no Estuário amazônico no estado do Amapá. Floresta, 3, 2007.

Ribeiro HMC, Ferreira FN. O Valor da Biodiversidade e o Mecanismo de Acesso e Repartição de Benefícios na Amazônia. In V Congresso Internacional de Direito Ambiental, 2018.

Rolim SG, Couto HTZD, Jesus RMD, França JT. Modelos volumétricos para a floresta nacional do Tapirapé-aquirí, Serra dos Carajás (PA). Acta Amazônica, 36, 107-114, 2006.

Rocha, AKP, Alves CP, Silva JN, Silva TGF, Leite MLMV, Cirino JB. Main ecosystems used as native pasture in Brazil: a review. Research, Society and Development, 9(10), 2020.

Rodrigues SR, dos Santos DRS, da Silva MM, Araujo A, Rocha CGS. Composição florística de fragmento de floresta secundária em áreas de proteção permanente do Igarapé Dispensa, no Projeto de Assentamento Assurini em Altamira, Pará. Biotemas, 33(1): 1-11, 2020.

Santos DL, Moraes JS, de Souza Araújo ZT, da Silva IR. Saberes tradicionais sobre plantas medicinais na conservação da biodiversidade amazônica. Ciências em Foco, 12 (1), 2019.

Schawartz G, Nascimento NA, Menezes AJEA. Estrutura populacional de espécies de interesse florestal não madeireiro no sudeste do Pará, Brasil. Amazônia: Ciência \& Desenvolvimento, 4 (7), 2008.

Silva CK. 2014. Potencial produtivo e manejo de dois produtos florestais não madeireiros no contexto Amazônico - o cipó-titica (Heteropsis spp.) e o óleo de copaíba (Copaifera spp). Tese (Doutorado em recursos florestais). Univeridade de São Paulo, 2014.

Silva M, Martins B, Nagy A, Maciel N. Políticas públicas do Brasil para produtos florestais não madeireiros. The Overarching Issues of the European Space- a strategic (re)positioning of environmental and socio-economic. Faculdade Letras Univ. Porto. 255-273, 2016.

Silva, L. C. Banco de Dados para Web. 1. ed. São Paulo: Érica, 2001. 
Silva EAS. 2006. Estudo dos óleos essenciais extraídos de resinas de espécies Protium spp. Tese de Doutorado (Mestre em Ciências). Universidade de São Paulo.

Silveira MI, Kern DC, Berreno JF, Costa JA, Costa ML COSTA. Um milênio de ocupações arqueológicas com manchas de terra preta em floresta na região de Carajás, Pará, Brasil. Boletim do Museu Paraense Emílio Goeldi. Ciências Naturais, 11(1): 11-31, 2016.

Silveira MI, Rodrigues MCLF, Oliveira ER, Losier LM. Arqueologia na floresta: contribuição metodológica da pesquisa na Floresta Nacional Tapirapé-Aquiri FLONATA, área do Salobo, Pará. R. Museu Arq. Etn, 25: 133-167, 2015.

Souza EC, Oliveira MR. Comparativo entre os bancos de dadosMYSQLe Mongobb:quandooMongoDBéindicado para o desenvolvimento de uma aplicação. Revista Interface Tecnológica, 16(2): 38-48, 2019.

Soares TS, Fiedler NC, Silva JA, Gasparini AJ. Produtos Florestais Não Madeireiros. Revista Científica Eletrônica de Engenharia Florestal, 11 (7), 2008.

Sousa RL, Almeida BB, Silva RP, Albuquerque LCS, Cordeiro YEM. Óleo de andiroba: extração, comercialização e usos tradicionais na comunidade Mamangal, Igarapé-Miri, Pará. Biodiversidade, 18(1), 2019.
Ter Steege et al. The discovery of the Amazonian tree flora with an updated checklist of all known tree taxa. Scientific Reports, 6, 1-15, 2016.

Théry NAM, Théry H. Carajás-Parauapebas: conflitos entre modelos de desenvolvimento na Amazônia Oriental. Praia Vermel ha, Rio de Janeiro, 19(2): 7388, 2009.

Vieira ALM, Mendonça MV, Albuquerque SS, Bezerra JR, Gondin R. Práticas agroecológicas no entorno da Floresta Nacional do Tapirapé-Aquiri. Cadernos de Agroecologia, 13(1), 2018.

Vries, P. Sampling Theory for Forest Inventory: A TeachYourself Course. Berlin, Heidelberg, New York, London, Paris, Tokyo: Springer-Verlag. 1986. 408p.

Ximenes LC, Gama JRV, Bezerra TG, Cruz GS Importância do açaí na renda mensal da comunidade quilombola Murumuru em Santarém, Pará. IberoAmericana de Ciências Ambientais, 11(2), 2020.

Yuyama, LKO et al. Caracterização físico-química do suco de açaí de Euterpe precatoria Mart. oriundo de diferentes ecossistemas amazônicos. Acta Amazônica, Manaus, 41(4): 545-552, 2011.

Zoghbi MGB, Siqueira JBG, Wolter ELA, Júnior OLP. Constituintes Químicos de Protium paniculatum (Burseraceae). Acta Amazônica, 24, 59-61, 1994.
Biodiversidade Brasileira - BioBrasil.
Edição Temática: PIBIC

n. 1,2022

http://www.icmbio.gov.br/revistaeletronica/index.php/BioBR

Biodiversidade Brasileira é uma publicação eletrônica científica do Instituto Chico Mendes de

Conservação da Biodiversidade (ICMBio) que tem como objetivo fomentar a discussão e a disseminação de experiências em conservação e manejo, com foco em unidades de conservação $e$ espécies ameaçadas.

ISSN: 2236-2886 